\title{
Control of photodissociation with the dynamic Stark effect induced by THz pulses
}

\author{
A. Tóth $\odot,,^{1, *}$ A. Csehi $\odot,{ }^{2,1}$ G. J. Halász, ${ }^{3}$ and Á. Vibók ${ }^{2,1, \dagger}$ \\ ${ }^{1}$ ELI-ALPS, ELI-HU Non-Profit Ltd., H-6720 Szeged, Dugonics tér 13, Hungary \\ ${ }^{2}$ Department of Theoretical Physics, University of Debrecen, H-4010 Debrecen, PO Box 5, Hungary \\ ${ }^{3}$ Department of Information Technology, University of Debrecen, H-4010 Debrecen, PO Box 12, Hungary
}

(Received 12 November 2019; accepted 19 February 2020; published 18 March 2020)

\begin{abstract}
We demonstrate how dynamic Stark control can be achieved on molecular photodissociation in the dipole limit, using single-cycle (full width at half maximum) laser pulses in the terahertz (THz) regime. As the laser-molecule interaction follows the instantaneous electric field through the permanent dipoles, the molecular potentials dynamically oscillate and so do the crossings between them. In this paper, we consider rotatingvibrating diatomic molecules (two-dimensional description) and reveal the interplay between the dissociating wave packet and the dynamically fluctuating crossing seam located in the configuration space of the molecules spanned by the $R$ vibrational and $\theta$ rotational coordinates. Our showcase example is the widely studied lithium fluoride molecule for which the two lowest $\Sigma$ states are nonadiabatically coupled at an avoided crossing (AC); furthermore a low-lying pure repulsive $\Pi$ state is energetically close. Optical pumping of the system in the ground state thus results in two dissociation channels: one indirect route via the AC in the ground $\Sigma$ state and one direct path in the $\Pi$ state. We show that applying $\mathrm{THz}$ control pulses with specific time delays relative to the pumping can significantly alter the population dynamics, as well as the kinetic energy and angular distribution of the photofragments.
\end{abstract}

DOI: 10.1103/PhysRevResearch.2.013338

\section{INTRODUCTION}

Thanks to the continuously developing laser technology, which has made it possible to generate light pulses with the length of a few femtoseconds or a few hundred attoseconds, quantum control techniques are among the most powerful tools of physics both in fundamental research and in practical applications. The field of research is rapidly growing and protocols have been adopted for studying different dynamical properties and features of molecules starting from small diatomics to really large polyatomic systems [1-27].

In recent years, efforts were invested to apply the dynamic Stark effect (DSE) for control of chemical dynamical processes [28-35]. It can be resonant or nonresonant depending on the applied light frequency. In the first situation the strong laser radiation fields can couple any two electronic states of the molecule due to the electric transition dipole moment and can also shape them. So-called light-induced nonadiabatic phenomena arise. Light-induced or "dressed" adiabatic potentials are formed, which incorporate the laser-molecule coupling effects. Numerous theoretical and experimental studies have demonstrated that the light-induced nonadiabatic phenomena (light-induced avoided crossings or light-induced

\footnotetext{
*Attila.Toth@eli-alps.hu

†Vibok@phys.unideb.hu

Published by the American Physical Society under the terms of the Creative Commons Attribution 4.0 International license. Further distribution of this work must maintain attribution to the author(s) and the published article's title, journal citation, and DOI.
}

conical intersections) have strong impact on the dynamical and spectroscopic properties of molecular systems [36-42]. In the second case, if the laser field is nonresonant with the energy difference of any two electronic states of the molecule, it still can have a significant dynamical effect due to shaping of the potential energy surfaces through the permanent dipole moments. This effect is very well studied in the literature as it provides a general tool for quantum control of atomic and molecular dynamical processes [28-34]. The dynamic Stark effect can be described either in the dipole or in the Raman limit. In the dipole limit the interaction follows the instantaneous electric field, whereas in the Raman limit (when the dipole approximation is symmetry forbidden) the interaction only follows the laser-pulse envelope [32].

In the present work our showcase example is the lithium fluoride molecule; therefore the control procedure relies on the dipole limit. The LiF molecule has already been studied in our former works $[43,44]$, where we discussed the role played by the lowest-lying $\Pi$ electronic state in the photodissociation of the molecule through the population dynamics, the angular distribution, and the kinetic energy release (KER) spectra of the photofragments. Describing appropriately the light-induced nonadiabatic phenomena the rotational degree of freedom has already been taken into account as the dynamical variable in those works. Although in the present work we focus on a different subject and control the dynamics by a single-cycle terahertz $(\mathrm{THz})$ laser pulse, the molecular rotation is also included in the numerical simulations so as to describe accurately the photodissociation process.

Recently, attention has been paid to control the dynamical and other properties of molecules by single-cycle THz pulses. 


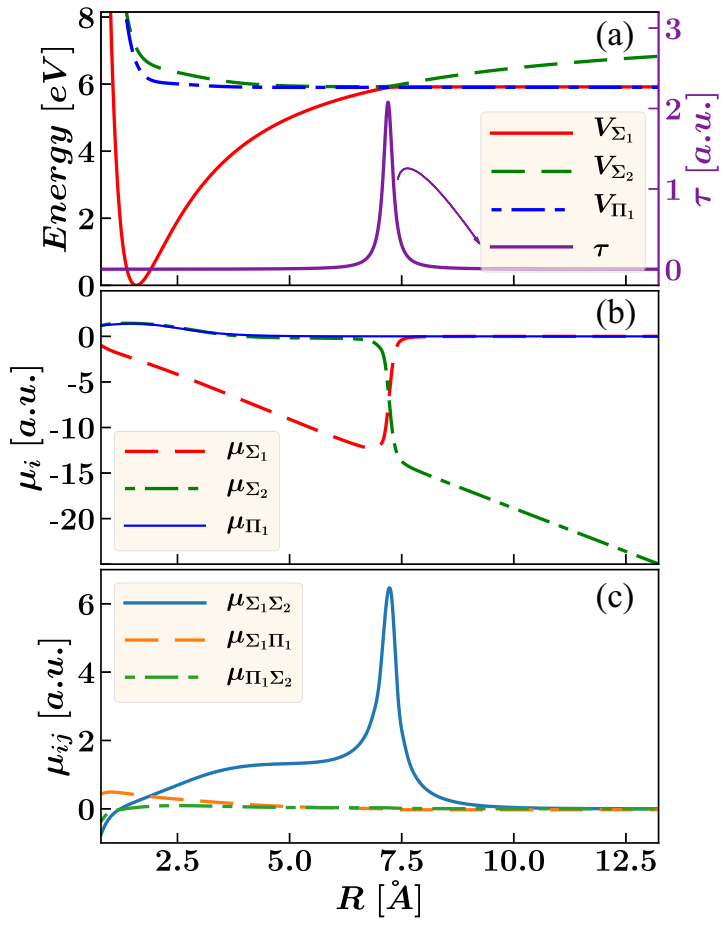

FIG. 1. (a) The lowest three adiabatic potential energy curves of the LiF molecule and the nonadiabatic coupling term $\tau(R)$ between the two $\Sigma$ states (scale is on the right side). (b) Permanent dipole moment functions of the three adiabatic electronic states. (c) The transition dipole moment functions between the different electronic states.

Fleischeret al. investigated both theoretically and experimentally the THz-induced molecular alignment in the gas phase using intense single-cycle $\mathrm{THz}$ pulses [45]. This group has also studied experimentally the decay of field-free rotational dynamics by terahertz-field-induced molecular orientation [46]. Kurosaki et al. proposed a theoretical control scheme of temporal wave packet separation for oriented molecules. By using linearly polarized single-cycle $\mathrm{THz}$ pulses they could separate the binary mixture of alkali halide isotopologues ${ }^{133} \mathrm{CsI}$ and ${ }^{135} \mathrm{CsI}$ [47]. Sub-one-cycle $\mathrm{THz}$ pulses were employed in the strategy suggested by Došlić [48] to achieve state-selective population transfer in the ACAC molecule.

In the present work we also wish to step in this direction of dynamic control exerted with $\mathrm{THz}$ radiation. In this lowfrequency range we aim to identify the main mechanisms that guide the system's response in such conditions by performing a thorough examination of the wave packet dynamics, and

$$
\hat{H}=\left(\begin{array}{ccc}
T & 0 & K \\
0 & T & 0 \\
-K & 0 & T
\end{array}\right)+\left(\begin{array}{c}
V_{\Sigma_{1}}-\mu_{\Sigma_{1}} \cos (\theta) E(t) \\
-\mu_{\Sigma_{1} \Pi_{1}} \sin (\theta) E(t) \\
-\mu_{\Sigma_{1} \Sigma_{2}} \cos (\theta) E(t)
\end{array}\right.
$$

Here, in the first term $T$ stands for the kinetic energy operator while $K$ is the intrinsic nonadiabatic coupling between states $\Sigma_{1}$ and $\Sigma_{2}$ at the avoided crossing. As we consider rotating- relate these effects to their fingerprints in various physical quantities, such as the kinetic energy release (KER) spectra and the angular distribution of the photofragments.

The paper is organized as follows: The working Hamiltonian and the computational details of the calculations are explained in Sec. II. In Sec. III, the results are presented and discussed. A summary and conclusions are given in the final section.

\section{THE PHYSICAL SITUATION AND METHODS}

Lithium fluoride along with the other alkali halides has been a popular testing ground for nonadiabatic dynamics during the photodissociation of these molecules due to the avoided crossing (AC) between their lowest lying ${ }^{1} \Sigma^{+}$electronic states. In our previous works on this system we showed that a realistic theoretical description must also include the first ${ }^{1} \Pi$ state $[43,44]$. Accordingly, in the present investigation we model the LiF molecule as a three-level system considering the $1^{1} \Sigma^{+}, 2^{1} \Sigma^{+}$, and $1^{1} \Pi$ electronic states, labeled throughout the paper as $\Sigma_{1}, \Sigma_{2}$, and $\Pi_{1}$. Their corresponding potential energy curves are presented in Fig. 1(a), along the intrinsic nonadiabatic coupling term $\left[\tau(R)=\left\langle\varphi_{\Sigma_{1}} \mid \frac{\partial}{\partial R} \varphi_{\Sigma_{2}}\right\rangle\right]$ linking the $\Sigma_{1}$ and $\Sigma_{2}$ states at the $\mathrm{AC}$ around $R \sim 7.2 \AA$. Panels (b) and (c) of Fig. 1 show the permanent $\left[\mu_{i}(R)=\right.$ $\left.-\left\langle\varphi_{i}\left|\sum_{k} r_{k}\right| \varphi_{i}\right\rangle\right]$ and the transition dipole moments $\left[\mu_{i j}(R)=\right.$ $\left.-\left\langle\varphi_{i}\left|\sum_{k} r_{k}\right| \varphi_{j}\right\rangle\right]$, respectively. An important feature of the transition dipole moments (TDMs) is that the one responsible for the $\Sigma-\Sigma$ transitions, i.e., $\vec{\mu}_{\Sigma_{1} \Sigma_{2}}$, is parallel with the molecular axis while the ones involving the $\Pi_{1}$ state are perpendicular.

Computation of the above electronic structure quantities of $\mathrm{LiF}$ have been carried out with the MOLPRO [49] program package at the MRCI/CAS(6/12)/aug-cc-pVQZ level of theory. In particular, the $\tau(R)$ has been computed by finite differences of the MRCI electronic wave functions. The number of active electrons and molecular orbitals in the individual irreducible representations of the $C_{2 v}$ point group were $A_{1} \rightarrow$ $2 / 5, B_{1} \rightarrow 2 / 3, B_{2} \rightarrow 2 / 3, A_{2} \rightarrow 0 / 1$. With these parameters, we achieved a good agreement with the results of other studies [50-52].

\section{A. Working Hamiltonian}

As stated above, in our previous works on the $\mathrm{LiF}$ we showed that for a realistic description of the dynamics of the molecule one should consider all three electronic states $\left(\Sigma_{1}\right.$, $\left.\Pi_{1}, \Sigma_{2}\right)$ in a theoretical calculation, and also its rotational motion. Accordingly, the time-dependent Hamiltonian employed in the present investigation reads

$$
\left.\begin{array}{cc}
-\mu_{\Sigma_{1} \Pi_{1}} \sin (\theta) E(t) & -\mu_{\Sigma_{1} \Sigma_{2}} \cos (\theta) E(t) \\
V_{\Pi_{1}}-\mu_{\Pi_{1}} \cos (\theta) E(t) & -\mu_{\Pi_{1} \Sigma 2} \sin (\theta) E(t) \\
-\mu_{\Pi_{1} \Sigma_{2}} \sin (\theta) E(t) & V_{\Sigma_{1}}-\mu_{\Sigma_{2}} \cos (\theta) E(t)
\end{array}\right) .
$$

vibrating molecules, the kinetic energy term is given by

$$
T(R, \theta)=-\frac{1}{2 M_{r}} \frac{\partial^{2}}{\partial R^{2}}+\frac{L_{\theta}^{2}}{2 M_{r} R^{2}},
$$




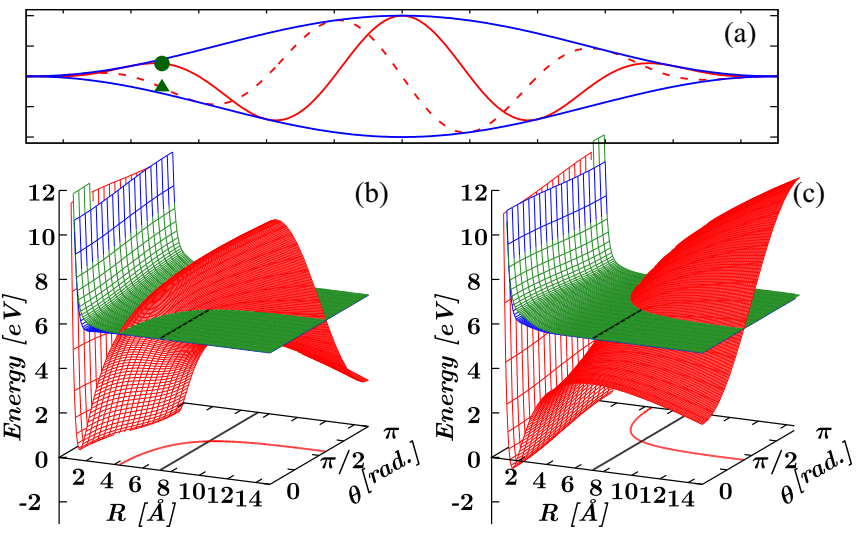

FIG. 2. (a) General form of the THz electric fields applied in the present work $\left(\hbar \omega_{c}=0.037 \mathrm{eV}, I_{c}=3.16 \times 10^{13} \mathrm{~W} / \mathrm{cm}^{2}\right)$. Two particular CEP cases of interest are presented $\left(\varphi_{c}=0\right.$ with solid red line and $\varphi_{c}=\pi / 2$ with dashed red line). The corresponding light-induced potential energy surfaces (LIPs) are shown in panel (b) for $\varphi_{c}=0$ and in panel (c) for $\varphi_{c}=\pi / 2$.

where $R$ is the internuclear distance and $\theta$ is the angle between the laser polarization direction and the molecular axis, i.e., the rotational coordinate. $M_{r}$ is the reduced mass, while $L_{\theta}$ is the angular momentum operator with $m=0$. For the nonadiabatic coupling operator we used an approximate form [53],

$$
K(R) \approx \frac{1}{2 M_{r}}\left[2 \tau(R) \frac{\partial}{\partial R}+\frac{\partial}{\partial R} \tau(R)\right],
$$

with $\tau$ being the nonadiabatic coupling term presented in Fig. 1(a).

The second term in the expression of $\hat{H}$ is the potential energy matrix including the coupling with the applied $E(t)$ laser field. As the different potential energy surfaces are dipole coupled, we restrict this light-matter interaction to the firstorder DSE, i.e., the dipole limit. Although the Hamiltonian of Eq. (1) was used throughout our calculations, it is easier to understand the system using the light-induced potentials (LIPs), in terms of which the potential energy matrix is diagonal [54]. They are presented in Fig. 2, and will serve a pivotal role in the interpretation of our results. Unless specified otherwise, atomic units with $e=m_{e}=\hbar=1$ are used throughout the article.

\section{B. The applied electric field}

In our calculations we used two linearly polarized (in the same direction) laser pulses, both of the form

$$
E(t, \varphi)=E_{0} f(t) \cos \left[\omega\left(t-t_{0}\right)+\varphi\right],
$$

with cosine-squared envelopes

$$
f(t)=\cos ^{2}\left(\frac{1.14372\left(t-t_{0}\right)}{\tau}\right),
$$

where $\tau$ is the full width at half maximum (FWHM) of the intensity profile. The dynamics was initiated by a $\tau_{p}=20 \mathrm{fs}$ long pump pulse, which also defined the origin of our time axis, that is, $t_{0 p}=0$. For all the results presented in this work, the energy of the pump was fixed to $\omega_{p}=6.94 \mathrm{eV}$, and its intensity to $I_{p}=5 \times 10^{12} \mathrm{~W} / \mathrm{cm}^{2}$.
The second one was a single-cycle $\mathrm{THz}$ pulse with $\omega_{c}=0.037 \mathrm{eV}$, with the corresponding pulse duration $\tau_{c}=$ $111.77 \mathrm{fs}$, and $I_{c}=3.16 \times 10^{13} \mathrm{~W} / \mathrm{cm}^{2}$. This control field is unable to produce transitions between the electronic states; however it alters the potential energy landscape of the molecule, which has a great impact on the outcome of the photodissociation process. The calculations were performed for two $\varphi_{c}$ carrier envelope phase (CEP) values, and used the time delay $\Delta t=t_{0 c}-t_{0 p}$ between the pulses as the "control knob" to steer the dynamics.

\section{Propagation of the wave packets}

The time-dependent Schrödinger equation (TDSE) that described the dynamics of the system was solved using the MCTDH (multiconfigurational time-dependent Hartree) method [55-57]. The vibrational degree of freedom $(R)$ was described by a sin-DVR primitive basis with $N_{R}$ basis elements distributed between 0.79 and $31.75 \AA$ for the internuclear separation. For the description of the rotational degree of freedom $(\theta)$ Legendre polynomials $\left\{P_{j}(\cos \theta)\right\}_{j=0,1,2, \ldots, N_{\theta}}$ were used. These primitive basis sets $(\chi)$ were employed to represent the single-particle functions $(\phi)$, which in turn were used to build up the nuclear wave function $(\psi)$ :

$$
\begin{aligned}
\phi_{j_{q}}^{(q)}(q, t) & =\sum_{l=1}^{N_{q}} c_{j_{q} l}^{(q)}(t) \chi_{l}^{(q)}(q), \quad q=R, \theta, \\
\psi(R, \theta, t) & =\sum_{j_{R}=1}^{n_{R}} \sum_{j_{\theta}=1}^{n_{\theta}} A_{j_{R}, j_{\theta}}(t) \phi_{j_{R}}^{(R)}(R, t) \phi_{j_{\theta}}^{(\theta)}(\theta, t) .
\end{aligned}
$$

In our numerical calculations $N_{R}=2048$ and $N_{\theta}=361$ primitive basis functions were used. In order to ensure the correct convergence of the propagations, on all adiabatic surfaces and for both degrees of freedom a set of $n_{R}=n_{\theta}=50$ singleparticle functions were used to build up the nuclear wave function of the system. This relatively high value was necessary as the $\mathrm{THz}$ control field induced a considerable amount of rotation.

\section{Calculated quantities}

The solutions of the TDSE were then used to calculate the populations of the employed electronic states [43], the kinetic energy release (KER) spectra, and the angular distribution of the molecular fragments [58]. The electronic state populations are obtained as

$$
\begin{aligned}
P_{i}(t) & =\left\langle\psi_{i}(R, \theta, t) \mid \psi_{i}(R, \theta, t)\right\rangle, \quad i \in\left\{\Sigma_{1}, \Pi_{1}, \Sigma_{2}\right\}, \\
& =\int_{0}^{\pi} d \theta \sin \theta \int_{0}^{\infty} d R \psi_{i}^{*}(R, \theta, t) \psi_{i}(R, \theta, t),
\end{aligned}
$$

where $\psi_{i}$ are the projections of the total nuclear wave function of Eq. (6) on the considered electronic states. The KER is calculated according to the following formula:

$$
P_{\mathrm{KER}}^{i}(E)=\int_{0}^{\infty} d t \int_{0}^{\infty} d t^{\prime}\left\langle\psi_{i}(t)|W| \psi_{i}\left(t^{\prime}\right)\right\rangle e^{-i E\left(t-t^{\prime}\right)},
$$

where $-i W$ is the complex absorbing potential (CAP) applied at the last $5.29 \AA$ of the grid related to the vibrational degree of freedom of each electronic state: $W=0.0000397$ 

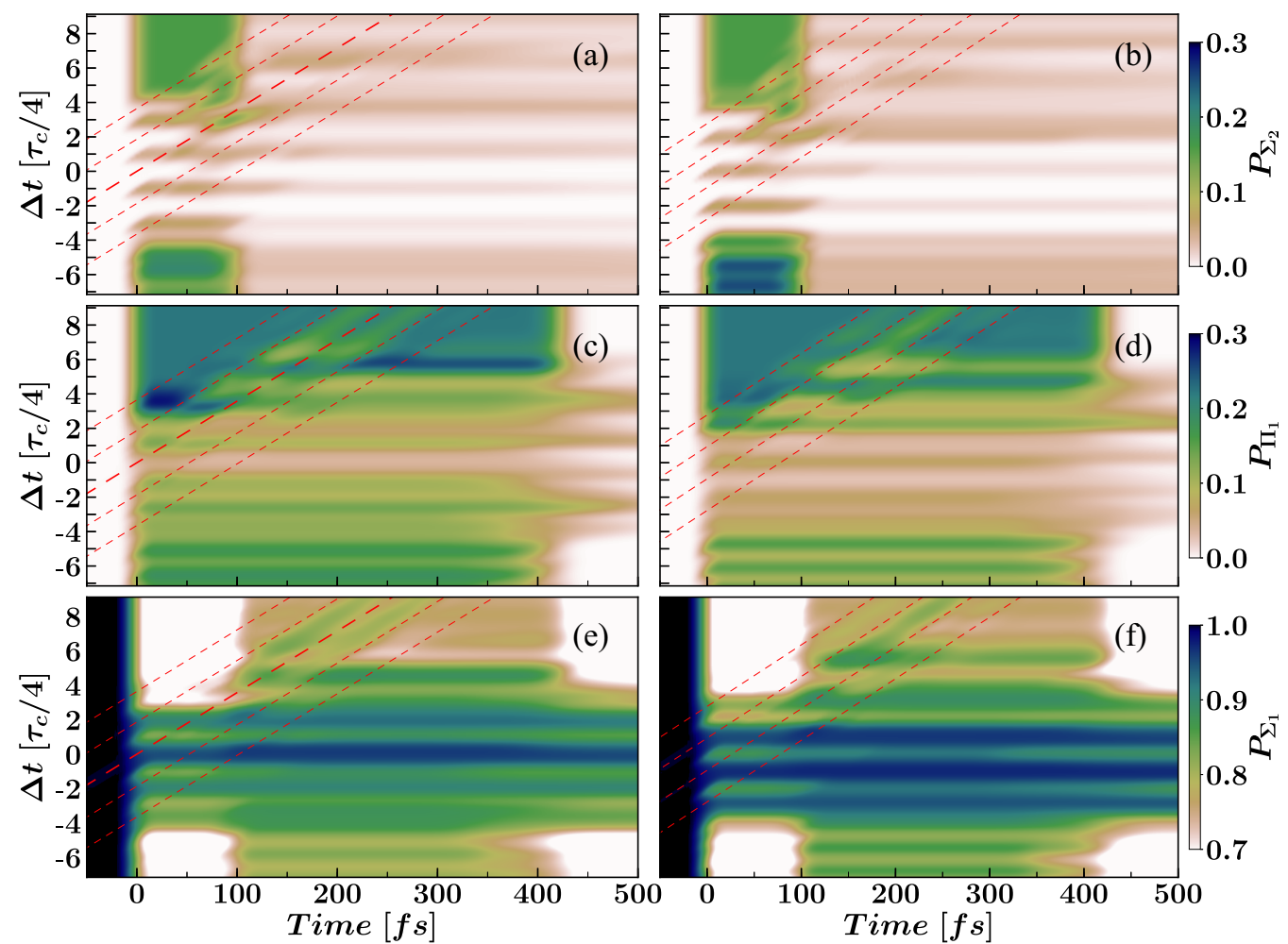

Time $[f s]$

FIG. 3. Time evolution (horizontal axis) of the populations in each electronic state $\left(\Sigma_{1}\right.$, bottom; $\Pi_{1}$, middle; $\Sigma_{2}$, upper panels in each column) calculated according to Eq. (7) for different $\Delta t$ delay times (vertical axis) for two different carrier-envelope phases: panels (a), (c), and (e) for $\varphi_{c}=0$ while panels (b), (d), and (f) for $\varphi_{c}=\pi / 2$. Transverse red lines mark the time moments when the electric field of the control pulse has minima/maxima.

$(R-26.46)^{3}$, if $R>26.46 \AA$. The angular distribution of the photofragments is given by

$$
P_{\text {ang }}^{i}\left(\theta_{j}\right)=\frac{1}{w_{j}} \int_{0}^{\infty} d t\left\langle\psi_{i}(t)\left|W_{\theta_{j}}\right| \psi_{i}(t)\right\rangle
$$

where $-i W_{\theta_{j}}$ is the projection of the CAP to a specific direction of the angular grid $\left(j=0, \ldots, N_{\theta}\right)$, and $w_{j}$ is the DVR weight associated to this grid point. In the last two equations the superscript $i$ stands for either $\Sigma_{1}$ or $\Pi_{1}$ as the molecule can dissociate on these two states.

\section{RESULTS AND DISCUSSION}

The dynamic Stark effect is usually examined as a function of the time delay between the pump and the Stark (control) pulse. We follow this tradition and start our investigations by looking at the evolution of the state populations changing the center of the control pulse from $t_{0 c}=-200$ fs to 255 fs. In the initial time moment $\left(t_{\text {init }}\right.$ preceding both the pump and the control pulse for all time delays) of the calculations we assumed randomly oriented molecules. The initial nuclear wave packet $\left[\Psi\left(t_{\text {init }}\right)\right]$ of this isotropic distribution was built from the rotational $J=0$ and the vibrational $v=0$ ground state of the $\Sigma_{1}$ electronic state. The results are presented in Figs. 3(a) and 3(b) for $\varphi_{c}=0$ and $\varphi_{c}=\pi / 2$, respectively. Here, the time delay is conveniently expressed in units of the control pulse period, $\tau_{c}=111.77 \mathrm{fs}$. Also, to help understand the data, red transverse dashed lines mark the time moments when the control field has an extremum (minimum or maximum).
The spacing between these lines is not $\tau_{c} / 2$ as the envelope of the pulse "pushes" the field extremum slightly toward the center of the pulse. From this figure it is clear that the choice of $\Delta t$ has a huge impact on the behavior of the system. This behavior differs however in a few key aspects from that found in the literature of the dynamic Stark effect. Those works almost exclusively describe the nonresonant dynamic Stark effect (NRDSE) in the moderately intensive (nonperturbative but nonionizing) regime and the Raman limit. As a consequence of the Stark shifted potentials the velocity with which the excited wave packet traverses the crossing region is altered, and according to the Landau-Zener formula [59] the branching ratio of the photofragments is modified. This control scheme is most pronounced when the Stark field is applied either during the pump process or when the wave packet is around the crossing point. If it comes before or after these time moments, the dynamics of the system remains unaffected.

The fundamental difference in the present work, as mentioned above, is that the electronic states are dipole coupled, meaning that the first-order DSE applies; hence the interaction follows the instantaneous electric field. Besides, the intensity of our control pulse, while still nonionizing, is relatively high, which combined with the first-order DSE leads to significant modifications of the potential surfaces, as illustrated by Fig. 2 . This leaves pronounced changes in the evolution of the state populations presented in Fig. 3, for all investigated time delays (in each case the nuclear time-dependent Schrödinger equation was propagated beyond $1 \mathrm{ps}$, but most of the dynamics 


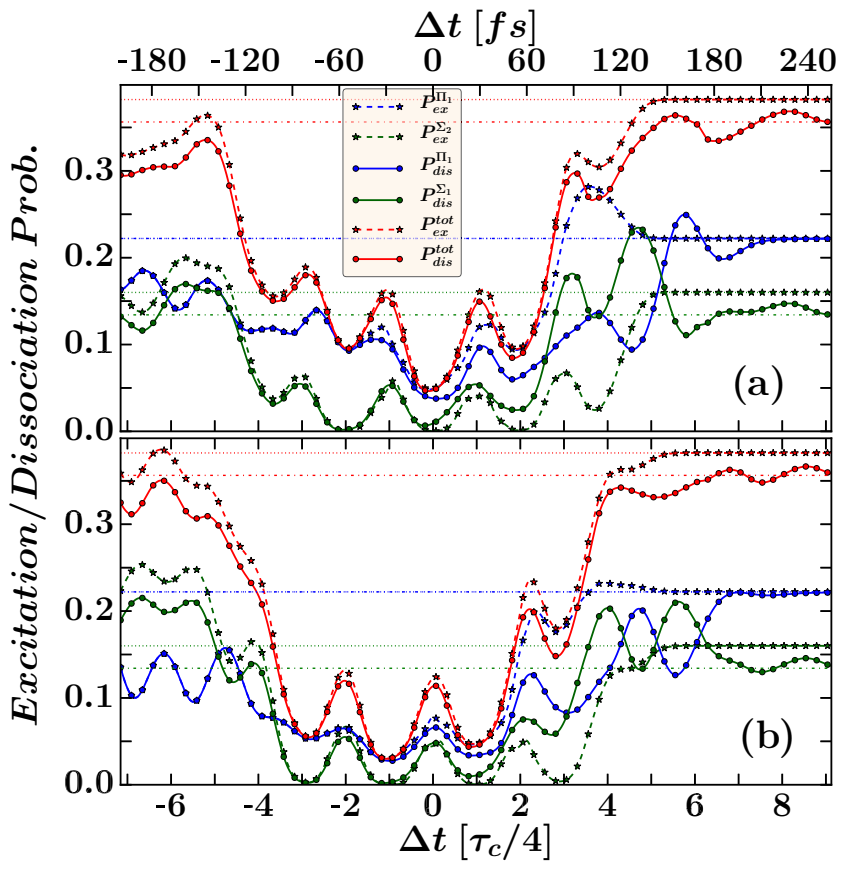

FIG. 4. Excited population in the $\Pi_{1}$ and $\Sigma_{2}$ states marked with blue and green dashed lines, and dissociation probability in the $\Pi_{1}$ and $\Sigma_{1}$ states marked with blue and green continuous lines. Red lines represent the sum of the excited and dissociated population of the above two channels. The control pulse carrier-envelope phase is (a) $\varphi_{c}=0$, (b) $\varphi_{c}=\pi / 2$.

ceased around $400 \mathrm{fs}$, when the dissociating wave packets reach the absorbing potential at the end of the numerical grid). The most striking feature is the suppression of the pump process when the two pulses overlap. In this interval the excited populations are not only decreased, but also show a modulation as a function of delay time, which resembles the periodicity of the control pulse. Interestingly, similar modulations are present even when the control pulse precedes the pump. The other important phenomenon that has to be noted is that after the dynamics is initiated there is usually a population transfer around the control field extremum, which in turn impacts the branching ratio between the dissociation channels $\mathrm{LiF} \rightarrow \mathrm{Li}+\mathrm{F}\left({ }^{2} P_{1 / 2}\right)$ and $\left.\mathrm{LiF} \rightarrow \mathrm{Li}+\mathrm{F}^{2} P_{3 / 2}\right)$ correlating to the $\Pi_{1}$ and $\Sigma_{1}$ states, respectively.

In order to better visualize the above findings, we present in Fig. 4 the excitation (dashed lines with stars) and dissociation (full curves with circles) probabilities of the different channels. Green and blue lines stand for quantities related to the $\Sigma_{2}$ (dissociation in $\Sigma_{1}$ ) and the $\Pi_{1}$ states, respectively, while red curves represent their sum. Also, horizontal dotted and dashed-dotted lines with the same color coding mark the excitation and dissociation probability of the system in the absence of the THz pulse. As $\Pi_{1}$ is a fully dissociative state the related horizontal blue lines overlap. It can be seen that after sufficiently long delay times $\left(\sim 5 \tau_{c} / 4\right)$ the populations pumped to the excited states converge to their values obtained in the control-free case. As mentioned above, during the overlap of the pump and the control fields the excitation efficiency is greatly reduced, and takes place in short bursts around the time moments when the instantaneous control field is zero. This is more pronounced for the $\Sigma_{2}$ state, which is practically unaffected by the pump when the electric field of the control pulse has an extremum. It is worth mentioning that in this $\Delta t$ range almost none of the excited population remains trapped in the $\Sigma_{2}$ state, as indicated by the proximity of the total excitation and dissociation curves. For smaller delay times, when the control pulse terminates before the pump is switched on, the excited population in the $\Pi_{1}$ state remains below its control-free value, while the one in the $\Sigma_{2}$ exceeds it. This is more prominent for the $\varphi_{c}=\pi / 2$ case.

The above detailed behavior of the pump process is rooted in our theoretical description, which is the consideration of the rotational degree of freedom. Traditional NRDSE control techniques rely on the use of infrared pulses, which are unable to produce any transitions in the investigated system (hence the name nonresonant). In contrast, we chose to work with a $\mathrm{THz}$ radiation, which while still unable to produce electronic transitions, induces rotational and vibrational excitations. These rotational excitations persist even after the control pulse is finished. This leads to the oscillation of a rotational wave packet in the ground electronic state, which is the cause of the modulations observed in the populations excited to the $\Sigma_{2}$ and $\Pi_{1}$ states for large negative delay times, as the pump pulse no longer encounters the original isotropic initial distribution. Moreover, the interference between the various components of this wave packet leads to the development of a nodal structure, which also manifests in the angular distribution of the photofragments. These angular distributions for the two considered carrier-envelope phases of the control pulse, $\varphi_{c}=0$ (left panel) and $\varphi_{c}=\pi / 2$ (right panel), are presented in Fig. 5 for the two distinct dissociation channels and also their sum. The figure shows that the dissociation occurs primarily along the (common) polarization axis of the employed laser pulses, and as Fig. 4 already suggested, mostly on the $\Sigma_{1}$ state. Considering the nature of the transition dipoles ( $\mu_{\Sigma_{1} \Sigma_{2}} / \mu_{\Sigma_{1} \Pi_{1}}$ is parallel/perpendicular to the molecular axis) this means that the $\mathrm{THz}$ pulse oriented the molecules along its polarization axis, and this orientation remained, or more precisely it was periodically partially revived, after the pulse ended.

The suppression of the pump process during the temporal overlap of the two laser pulses can be best understood based on the light-induced potentials presented in Fig. 2. In order to have an efficient population transfer between two dipolecoupled electronic surfaces, two conditions have to be met: the coupling radiation has to be resonant for a given region $[(R, \theta)$ in our $2 \mathrm{D}$ case] of the involved surfaces, and these regions need to be populated. As we saw earlier, the $\mathrm{THz}$ control pulse induces a rotational excitation of the system. Moreover, as the $\mathrm{LiF}$ is a polar molecule, the control field orients the molecule instead of aligning it. In the LIP picture this manifests in the deformation of the potential surfaces along the $\theta$ coordinate: for a given internuclear distance, the potential energy surface (PES) ascends or descends compared to its field-free position along the $\theta$ direction due to the $\mu_{i} \cos (\theta) E(t)$ term of the Hamiltonian. In other words, a potential well forms around $\theta=\{0, \pi\}$ periodically. For our initial isotropic distribution in the ground state this means a periodic concentration in these potential wells, i.e., up or down orientation of the molecules. Another important factor is 


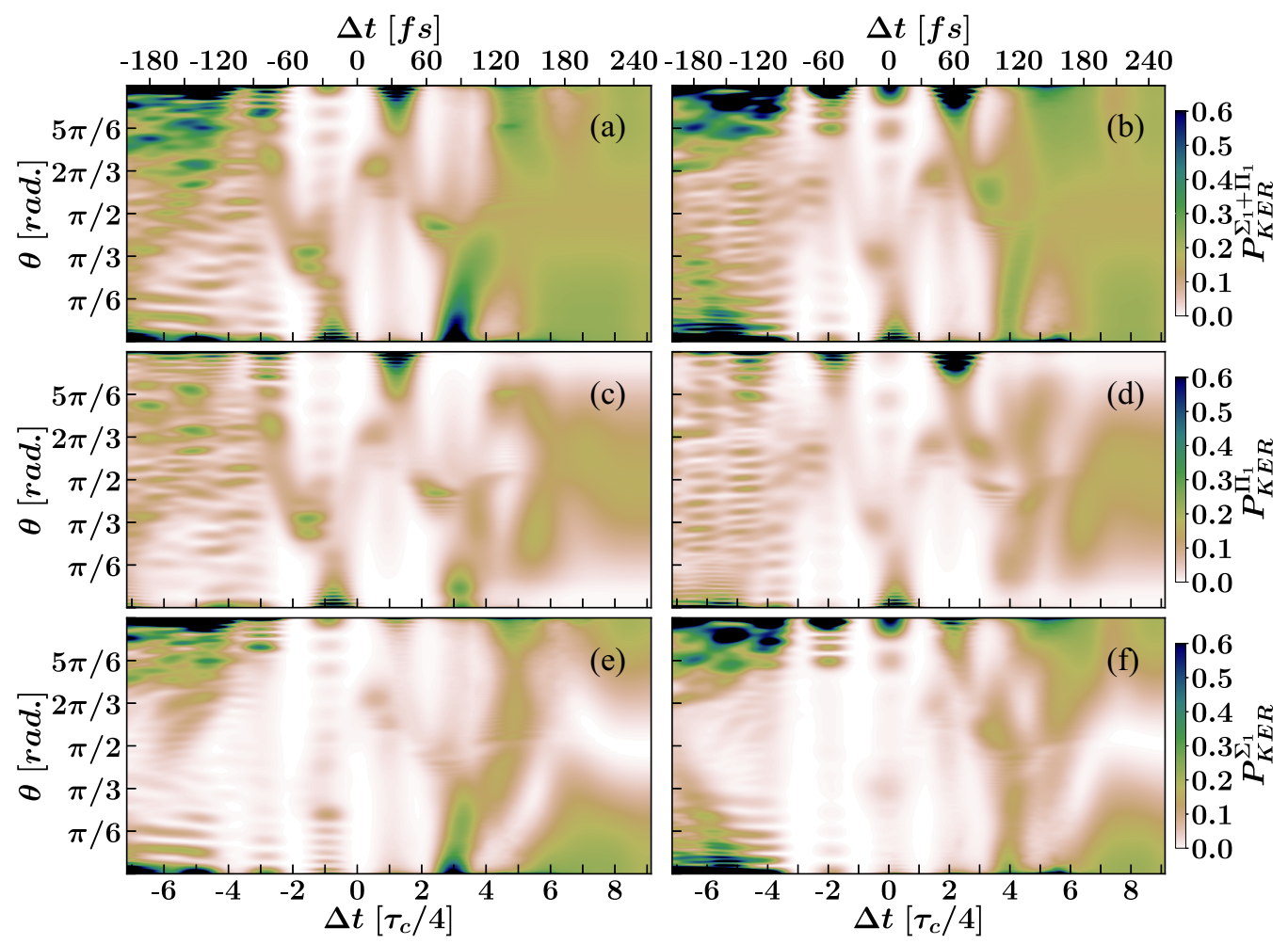

FIG. 5. Angular distribution (vertical axis) of the photofragments in the $\Sigma_{1}$ (bottom) and $\Pi_{1}$ (middle) electronic states, and the sum of the two channels (top) as a function of time delay (horizontal axis) between the pump and the control pulses for the two investigated carrier-envelope phases: panels (a), (c), and (e) for $\varphi_{c}=0$ while panels (b), (d), and (f) for $\varphi_{c}=\pi / 2$. The distributions associated with each delay time were calculated conforming to Eq. (9).

that the permanent dipoles of the excited states have opposite signs compared to the ground state permanent dipole moment in the Franck-Condon region, which means that they are displaced in the opposite direction than $\Sigma_{1}$. Accordingly, when the molecules are oriented either up or down, the detuning between the $\Sigma$ states exceeds the pump energy and instead of an increased excited population we end up with none. The condition of population transfer to $\Sigma_{2}$ exists only in a short time window around the time moments when the control field is zero which again reduces the pump efficiency.

The situation of the $\Pi_{1}$ state is more interesting. The fundamental difference here is that the TDM with the ground state is perpendicular to the molecular axis [43]. This means that the two states are coupled in the region where the control field least distorts the potential surfaces (see again Fig. 2). Due to these facts, intuitively one would expect most of the dissociating fragments to be detected perpendicular to the laser polarization direction; however this is not the case. Having in mind that the pump energy was tuned to the $\Sigma_{1}-\Sigma_{2}$ transition, it is easy to see that the resonance condition between $\Sigma_{1}$ and $\Pi_{1}$ is shifting along the $\theta$ coordinate as the PESs swing under the action of the control pulse. This movement of the resonance point can be identified in the angular distributions $P_{\text {ang }}^{\Pi_{1}}$ in the $\Delta t \in\left[-3 \tau_{c}, 5 \tau_{c}\right]$ delay time interval, although it is not a one-to-one correspondence, as the excited wave packet is slightly (due to the considerably smaller $\mu_{\pi_{1}}$ than $\mu_{\Sigma_{1}}$ ) rotated on the distorted PES. More surprising is that in this interval the molecules dissociate with highest probability along the laser polarization direction. This can be understood in light of the wave packet dynamics on the LIPs described earlier. As we saw, the control pulse orients the molecules up or down. Due to the fact that $\Pi_{1}$ lays lower in energy than $\Sigma_{2}$, the resonance condition with the ground state along the polarization axis is achieved before the control field changes its sign (hence, the peaks are shifted from the zero control field moments). Accordingly, most of the ground state population is still concentrated in this (up or down) region, which results in a higher transition probability to $\Pi_{1}$ despite the reduced coupling. Moreover, as the field changes sign, the excited states develop potential wells in the direction which previously $\Sigma_{1}$ had (up or down), which results in the rotation of the $\Pi_{1}$ wave packet toward the pump-forbidden $\theta=\{0, \pi\}$ direction. This in turn leads to the development of the interference structures observable in the angular distribution [44]. If the control pulse is applied after the system is pumped but before the excited wave packets reach the AC region the angular distributions are more structured owing to the previously mentioned population transfer between the various states. This is most evident by the appearance of dissociating fragments around the perpendicular direction on $\Sigma_{1}$ accompanied by a reduced dissociation probability at the same time delays on $\Pi_{1}$. Finally, if the control pulse is turned on after the excited wave packet traverses the $\mathrm{AC}$ region, the angular distributions converge to their usual control-free dipole shapes.

The Stark deformation of the potential surfaces depends on a number of factors: control field intensity, internuclear distance dependence of the permanent dipoles, and orientation of the molecules. In addition, the used control field changes sign 


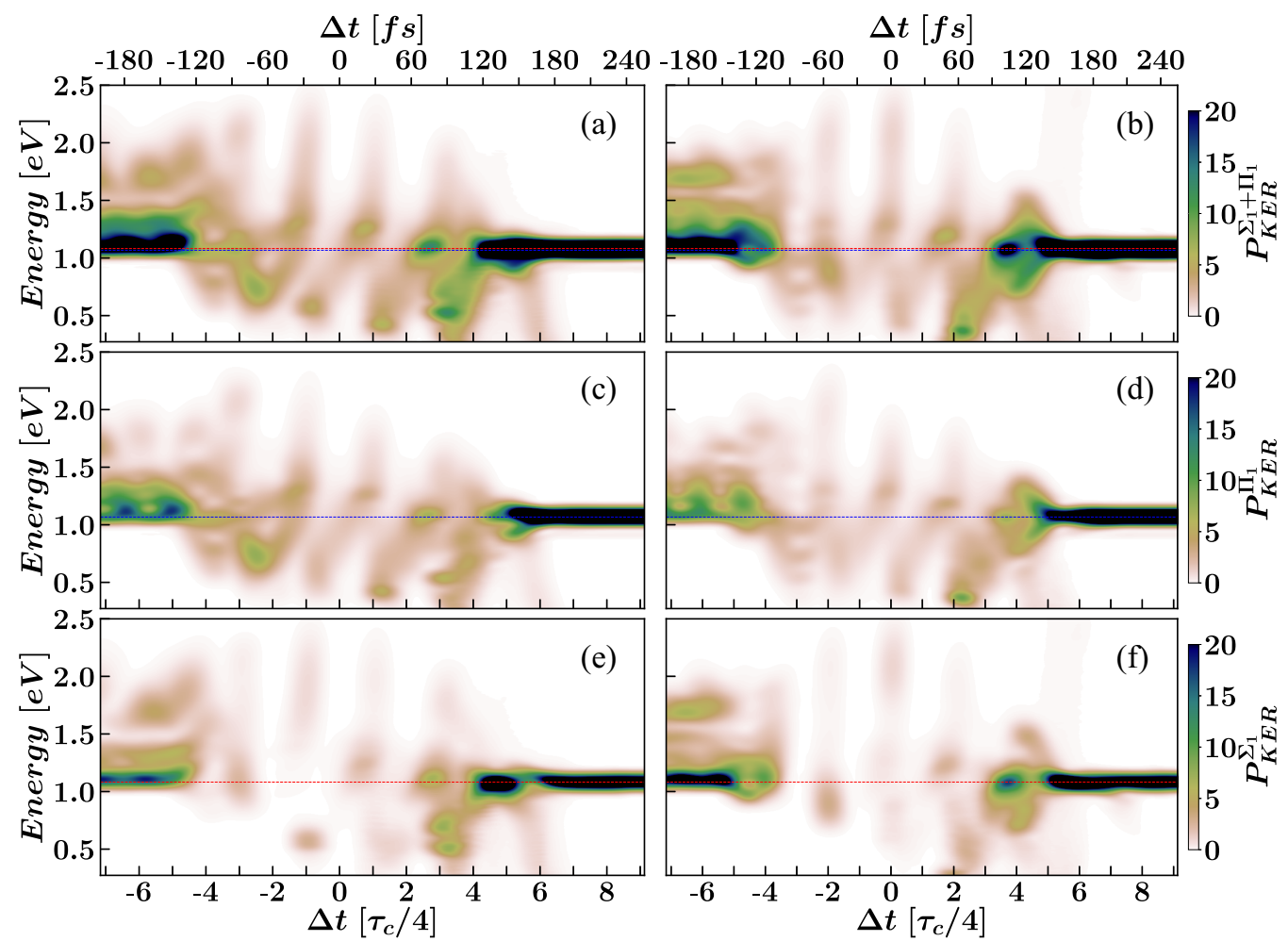

FIG. 6. Kinetic energy release distribution (vertical axis) of the photofragments in the $\Sigma_{1}$ (bottom) and $\Pi_{1}$ (middle) electronic states, and the sum of the two channels (top) as a function of time delay (horizontal axis) between the pump and the control pulses for the two investigated carrier-envelope phases: panels (a), (c), and (e) for $\varphi_{c}=0$ while panels (b), (d), and (f) for $\varphi_{c}=\pi / 2$. The distributions associated with each delay time were calculated conforming to Eq. (8).

a number of times, which leads to an intricate wave packet dynamics. Following this dynamics for each considered time delay is a cumbersome task which extends beyond the purpose of the present work. However, the main mechanisms shaping the response of the system toward the interaction with the control field can be identified.

The effect of the control pulse on the excitation process was detailed above based on the angular distribution of the photofragments. Complementary information is provided by the kinetic energy release spectra of the dissociation products. These are presented in Fig. 6 in an arrangement similar to the angular distributions of Fig. 5. Red and blue horizontal dashed lines mark the center of the KER spectra (Lorentzian shaped due to the single-photon pump process) in a pump-only scenario in the $\Sigma_{1}$ and $\Pi_{1}$ states, respectively. These results consolidate what we observed earlier. For large negative time delays we see that higher energies are present in the spectra, indicating that the molecules were rovibrationally excited in the ground state before the pump-induced transitions to the excited electronic states. In the other extreme, for large positive delays, just as with the angular distributions, the KER spectra also converge to their control-free value. In between, when the control pulse is present while the excited wave packets reach the AC, the spectra are smeared both to higher and lower energies than in the control-free case. This is the result of two processes.

First of all, as the PESs are fluctuating under the action of the control pulse, the potential energy of the dissociating wave packets is altered, which ultimately translates to modifications of the final kinetic energy of the photofragments. Whether it is increased or decreased depends on which region $(\theta<\pi / 2$ or $\theta>\pi / 2$ ) of the excited surface was the population placed on, and the phase of the control pulse (direction of the electric field). The magnitude of the energy shift follows the $\theta$ dependence of the PES modulations (strongest for the direction parallel with the laser field and none in the perpendicular direction). This is reflected in the fact that the smallest KER values are obtained whenever the population is pumped in the direction of the field, where as we saw while discussing the angular distributions, the excited surfaces develop potential wells.

The second process is the above-mentioned population transfers observed in the $\sim \Delta t \in\left[0,7 \tau_{c} / 4\right]$ interval. This can also be attributed to the dynamically changing potential surfaces. Earlier works found in the literature [54,60] pointed out that in a diabatic picture the dynamically Stark shifted potentials also imply that the position of the crossing between the nonadiabatically coupled $\Sigma_{1}$ and $\Sigma_{2}$ states of $\mathrm{LiF}$ also changes as a function of time. This is illustrated in Fig. 2, where the continuous black lines in the $R-\theta$ plane mark the position of the intrinsic $\mathrm{AC}$ in the control-free case, while the red curve indicates the crossing between the light-induced potentials at a given time moment during the action of the control pulse, marked by a green circle and triangle in the plot of the electric fields of Fig. 2(a). Moreover, in our three-state description a new light-induced crossing emerges between the $\Sigma_{1}$ and $\Pi_{1}$ states (due to the proximity of $\Sigma_{2}$ and $\Pi_{1}$ this crossing is close to the $\Sigma_{1}-\Sigma_{2}$ one, and for clarity, only the latter one is plotted 
in the figures). It is obvious that the instantaneous field intensity determines how much the dynamical crossing is shifted from its field-free position. Furthermore, we pointed out above that the LIPs swing along the $\theta$ coordinate, which leads to the $\theta$ dependence of the light-induced crossings. Whenever the dissociating wave packet encounters these dynamically shifting LIP intersections it bifurcates, leading to population transfers between the involved surfaces. This takes place when the crossing is shifted to smaller internuclear distances, i.e., where $\Sigma_{1}$ is lifted upward. As a result the population transferred to this state encounters a potential barrier and loses some of its kinetic energy before being transferred back to the excited states during the descending edge of the control pulse peak, when the crossing moves from smaller to larger internuclear distances.

However, it is hard to distinguish the above two effects in the KER spectra; this later one is more prominent in the state populations of Fig. 3. Here, for positive time delays the control field is strong enough to shift the crossings in the path of the dissociating wave packets. As the $\Pi_{1}$ PES lies lower in energy, it is encountered first by the ascending $\Sigma_{1}$ surface, and part of the population is transferred. Immediately afterward the $\Sigma_{1}-\Sigma_{2}$ bifurcation occurs, whereupon part of the population initially pumped to the $\Pi_{1}$ state gets in $\Sigma_{1}$. On the descending edge of the pulse peak the situation is reversed, and due to the stronger $\mu_{\Sigma_{1} \Sigma_{2}}$ TDM most of the dissociating population in $\Sigma_{1}$ is transferred to $\Sigma_{2}$ and only a small amount returns to $\Pi_{1}$. Accordingly, the control pulse unidirectionally modifies the branching ratio of the dissociation products favoring the $\Sigma_{1}$ channel. This effect seems to be the strongest around the highest central control field peak for both investigated CEP values; however this is somewhat hard to assess, as in this delay time region the initial excited populations are not the same due to the overlap of the two pulses.

Finally, the control pulse modifies not only the branching ratio of the dissociation channels, but also alters the amount of population temporally trapped on the bound $\Sigma_{2}$ state. This effect is best observed in the modulation of the difference between the total excitation and dissociation probabilities of Fig. 4 (red curves). This happens for larger delay times, when the trailing edge of the control pulse Stark-shifts the potential surfaces only when the dissociating wave packets are already in the neighborhood of the AC leading to the well-studied modulations [12,30,32] of the population transfer between the nonadiabatically coupled surfaces.

\section{CONCLUSIONS}

In this work we have investigated the effect of a $\mathrm{THz}$ control pulse on the photodissociation process of the $\mathrm{LiF}$ molecule. Besides the vibrational degree of freedom our description also incorporated the rotational motion of the molecule. For the employed control frequency we saw that this choice is indispensable for a realistic description of the system dynamics. The most prominent effect of the control pulse was the suppression of the excitation during the temporal overlap of the employed pulses. This is because the $\mathrm{THz}$ pulse drove the system out of resonance with respect to the pump energy, due to its significant permanent dipole, leading to intermittent population transfers to the excited states around the zero points of the control field. We have seen that the Stark fluctuations of the potential surfaces lead to the orientation of the initially unaligned molecules along the control polarization axis. Besides altering the pump efficiency, the control pulse induced dynamically shifting crossings between the light-induced potentials. As the dissociating wave packets encountered these crossings population transfers occurred, which led to a modulation of the $\Sigma_{1} / \Pi_{1}$ dissociation branching ratio in favor of the former.

Calculations were performed for two different carrier envelope phases. Changing it altered the timing of the population transfers, but otherwise did not impact the outcome of the dissociation process significantly.

The present investigation provided us with a solid understanding of the system dynamics, which is the basis of further optimizations of control strategies. As the intensity of the control pulse dictates the magnitude of the PES deformations while the frequency its timing, we wish to explore in further works how these parameters might be tuned to obtain stateselective excitation or a more pronounced modulation of the dissociation branching ratio.

\section{ACKNOWLEDGMENTS}

This research was supported by the EU-funded Hungarian Grant No. EFOP-3.6.2-16-2017-00005, and the ELI-ALPS project GINOP 2.3.6-15-2015-00001. We are grateful to NKFIH for support (Grant No. K128396).
[1] S. A. Rice and M. Zhao, Optical Control of Molecular Dynamics, Baker Lecture Series (John Wiley \& Sons, Inc., New Jersey, 2000).

[2] V. S. Letokhov, Laser Control of Atoms and Molecules (Oxford University Press, Oxford, 2007).

[3] P. W. Brumer and M. Shapiro, Principles of the Quantum Control of Molecular Processes (John Wiley \& Sons, Inc., New Jersey, 2003).

[4] H. Rabitz, R. de Vivie-Riedle, M. Motzkus, and K. Kompa, Science 288, 824 (2000).

[5] M. Wollenhaupt, V. Engel, and T. Baumert, Annu. Rev. Phys. Chem. 56, 25 (2005).
[6] A. Auger, A. Ben Haj-Yedder, E. Cances, C. Le Bris, C. M. Dion, A. Keller, and O. Atabek, Math. Models Methods Appl. Sci. 12, 1281 (2002).

[7] C. M. Dion, A. Ben Haj-Yedder, E. Cancès, C. Le Bris, A. Keller, and O. Atabek, Phys. Rev. A 65, 063408 (2002).

[8] C. M. Dion, A. Keller, and O. Atabek, Phys. Rev. A 72, 023402 (2005).

[9] Y. Arasaki, K. Takatsuka, K. Wang, and V. McKoy, J. Chem. Phys. 119, 7913 (2003).

[10] Y. Arasaki, S. Scheit, and K. Takatsuka, J. Chem. Phys. 138, 161103 (2013) 
[11] Y. Arasaki, Y. Mizuno, S. Scheit, and K. Takatsuka, J. Chem. Phys. 144, 044107 (2016).

[12] G. A. Worth and G. W. Richings, Annu. Rep. Prog. Chem. Sect. C: Phys. Chem. 109, 113 (2013).

[13] H. Stapelfeldt and T. Seideman, Rev. Mod. Phys. 75, 543 (2003).

[14] R. J. Gordon and T. Seideman, in Advances in Multi-Photon Processes and Spectroscopy (World Scientific, Singapore, 2016), Chap. 1, Vol. 23, pp. 1-54.

[15] C. Trallero-Herrero, D. Cardoza, T. C. Weinacht, and J. L. Cohen, Phys. Rev. A 71, 013423 (2005).

[16] M. Kotur, T. Weinacht, B. J. Pearson, and S. Matsika, J. Chem. Phys. 130, 134311 (2009).

[17] D. Geißler, P. Marquetand, J. González-Vázquez, L. González, T. Rozgonyi, and T. Weinacht, J. Phys. Chem. A 116, 11434 (2012).

[18] E. Persson, J. Burgdörfer, and S. Gräfe, New J. Phys. 11, 105035 (2009).

[19] L. Levin, W. Skomorowski, L. Rybak, R. Kosloff, C. P. Koch, and Z. Amitay, Phys. Rev. Lett. 114, 233003 (2015).

[20] R. E. Goetz, A. Karamatskou, R. Santra, and C. P. Koch, Phys. Rev. A 93, 013413 (2016).

[21] C. P. Koch, L. Mikhail, and D. Sugny, Rev. Mod. Phys. 91, 035005 (2019).

[22] I. R. Solá, B. Y. Chang, J. Santamaría, V. S. Malinovsky, and J. L. Krause, Phys. Rev. Lett. 85, 4241 (2000).

[23] B. Y. Chang, H. Rabitz, and I. R. Sola, Phys. Rev. A 68, 031402(R) (2003).

[24] B. Y. Chang, S. Shin, A. Palacios, F. Martín, and I. R. Sola, J. Chem. Phys. 139, 084306 (2013).

[25] B. Y. Chang, S. Shin, V. Malinovsky, and I. R. Sola, J. Phys. B: At. Mol. Opt. Phys. 48, 174005 (2015).

[26] B. Y. Chang, S. Shin, A. Palacios, F. Martín, and I. R. Sola, J. Phys. B: At. Mol. Opt. Phys. 48, 043001 (2015).

[27] R. Chamakhi, M. Telmini, O. Atabek, and E. Charron, Phys. Rev. A 100, 033402 (2019).

[28] B. J. Sussman, M. Y. Ivanov, and A. Stolow, Phys. Rev. A 71, 051401(R) (2005).

[29] B. J. Sussman, J. G. Underwood, R. Lausten, M. Y. Ivanov, and A. Stolow, Phys. Rev. A 73, 053403 (2006).

[30] B. J. Sussman, D. Townsend, M. Y. Ivanov, and A. Stolow, Science 314, 278 (2006).

[31] B. J. Sussman, Am. J. Phys. 79, 477 (2011).

[32] D. Townsend, B. J. Sussman, and A. Stolow, J. Phys. Chem. A 115, 357 (2011).

[33] Y.-C. Han, K.-J. Yuan, W.-H. Hu, and S.-L. Cong, J. Chem. Phys. 130, 044308 (2009).

[34] Y. Liu, Y. Liu, and Q. Gong, Phys. Rev. A 85, 023406 (2012).

[35] B. Mignolet, B. F. E. Curchod, F. Remacle, and T. J. Martínez, J. Phys. Chem. Lett. 10, 742 (2019).
[36] G. J. Halász, M. Šindelka, N. Moiseyev, L. S. Cederbaum, and Á. Vibók, J. Phys. Chem. A 116, 2636 (2012).

[37] G. J. Halász, Á. Vibók, and L. S. Cederbaum, J. Phys. Chem. Lett. 6, 348 (2015).

[38] A. Csehi, G. J. Halász, L. S. Cederbaum, and Á. Vibók, Faraday Discuss. 194, 479 (2016).

[39] A. Csehi, G. J. Halász, L. S. Cederbaum, and Á. Vibók, Phys. Chem. Chem. Phys. 19, 19656 (2017).

[40] T. Szidarovszky, G. J. Halász, A. G. Császár, L. S. Cederbaum, and Á. Vibók, J. Phys. Chem. Lett. 9, 2739 (2018).

[41] T. Szidarovszky, A. G. Császár, G. J. Halász, and Á. Vibók, Phys. Rev. A 100, 033414 (2019).

[42] A. Natan, M. R. Ware, V. S. Prabhudesai, U. Lev, B. D. Bruner, O. Heber, and P. H. Bucksbaum, Phys. Rev. Lett. 116, 143004 (2016).

[43] A. Tóth, P. Badankó, G. J. Halász, Á. Vibók, and A. Csehi, Chem. Phys. 515, 418 (2018).

[44] A. Tóth, A. Csehi, G. J. Halász, and Á. Vibók, Phys. Rev. A 99, 043424 (2019).

[45] S. Fleischer, Y. Zhou, R. W. Field, and K. A. Nelson, Phys. Rev. Lett. 107, 163603 (2011).

[46] R. Damari, D. Rosenberg, and S. Fleischer, Phys. Rev. Lett. 119, 033002 (2017).

[47] Y. Kurosaki, H. Akagi, and K. Yokoyama, Phys. Rev. A 90, 043407 (2014).

[48] N. Došlić, J. Phys. Chem. A 110, 12400 (2006).

[49] H.-J. Werner, P. J. Knowles, G. Knizia, R. Manby Frederick, and M. Schütz, MOLPRO, Version 2015.1, A Package of ab initio Programs, 2015.

[50] A. J. C. Varandas, J. Chem. Phys. 131, 124128 (2009).

[51] J. F. Triana, D. Peláez, and J. L. Sanz-Vicario, J. Phys. Chem. A 122, 2266 (2018).

[52] J. F. Triana and J. L. Sanz-Vicario, Phys. Rev. Lett. 122, 063603 (2019).

[53] A. Hofmann and R. de Vivie-Riedle, Chem. Phys. Lett. 346, 299 (2001).

[54] S. Scheit, Y. Arasaki, and K. Takatsuka, J. Phys. Chem. A 116, 2644 (2012).

[55] H.-D. Meyer, U. Manthe, and L. Cederbaum, Chem. Phys. Lett. 165, 73 (1990).

[56] M. Beck, A. Jäckle, G. Worth, and H.-D. Meyer, Phys. Rep. 324, 1 (2000).

[57] G. A. Worth, M. H. Beck, A. Jäckle, and H.-D. Meyer, The MCTDH Package: Version 8.2, University of Heidelberg, 2000. H.-D. Meyer, The MCTDH Package: Version 8.3 and 8.4, University of Heidelberg, 2002 and 2007, http://mctdh.uni-hd.de.

[58] G. J. Halász, A. Csehi, Á. Vibók, and L. S. Cederbaum, J. Phys. Chem. A 118, 11908 (2014).

[59] C. Wittig, J. Phys. Chem. B 109, 8428 (2005).

[60] S. Scheit, Y. Arasaki, and K. Takatsuka, J. Chem. Phys. 140, 244115 (2014). 